\title{
Algorithms for Pattern Recognition in Images of Cell Cultures
}

\author{
Joyce Martins Mendes / Nathalia L.V. Peixoto / Francisco Javier Ramirez Fernandez \\ E-mail: joycemm@lme.usp.br; jramirez@lme.usp.br \\ Av. Prof. Luciano Gualberto, travessa 3, 158 - CEP: 05508-900- SP \\ Fone: +55 11 818-5310 FAX:+55 11 818-5585 - São Paulo- Brasil \\ Web: http://sim.lme.usp.br
}

\begin{abstract}
Several applications of silicon microstructures in areas such as neurobiology and electrophysiology have been stimulating the development of microsystems with the objective of mechanical support to monitor and control several parameters in cell cultures. In this work a multi-microelectrode arrays was fabricated over a glass plate to obtain the growth of neuronal cell monitoring their behavior during cell development. To identify the neuron core and axon an approach for implementation of edge detectors algorithms associated to images is described. The necessity of efficient and reliable algorithms for image processing and interpretation is justified by its large field of applications in several areas as well as medicine, robotics, cellular biology, computational vision and pattern recognition. In this work, it is investigated the adequacy of some edge detectors algorithms such as Canny, Marr-Hildreth. Some alterations in those methods are propose to improve the identification of both cell core and axonal growth measure. We compare the operator to edge detector proposed by Canny, Marr-Hildreth operator and application of Hough Transform. For evaluation of algorithms adaptations, we developed a method for automatic cell segmentation and measurement. Our goal is to find a set of parameters defining the location of the objects to compare the original and processed images.
\end{abstract}

Key Words. Technical vision, processing and recognition of image

\section{Introduction}

Several applications of silicon microstructures in areas such as neurobiology and electrophysiology have been stimulating the development of microsystems with the objective of mechanical support to monitor and control several parameters in cell cultures. In this work a multi-microelectrode arrays was fabricated over a glass plate to obtain the growth of neuronal cell monitoring their behavior during cell development.

In this cases edge detection is a appropriate operation to identify the neuron core and axon. The reason for this is that edges form the outline of an object. An edge is the boundary between an object and the background and indicates the boundary between overlapping objects. This means that if the edges in an image can be identified accurately, the objects characterized by these edges can be located and properties like shape, perimeter, in some cases even distance, can be measured. Since technical vision involves the identification and classification of objects in an image, edge detection is an essential tool.

Technically, edges are places in the image with strong intensity contrast. Since edge often occur at image locations representing objet boundaries, edge detection is extensively used in image segmentation when we want to divide the image into areas corresponding to different objects. Representing an image by its edge has the further advantage that the amount of data is reduced significantly retaining most of the image information. [1-7]

A succinct review of the more significant approaches of edge detectors available today permit to indicate the next techniques as the most representative way to pre-process an image. For example, Gregson[8]uses a combination of contrast thresholding and analysis of direction dispersion to find edges. Baker and Binfor[9], and Ohta and Kanade[10] label peaks in the magnitude of the first derivative of intensity profile along a scan-lines as features points formatting. The Haralick[11] edge operator employs a step-edge detector based on the second directional derivative. Other popular gradient edge detector are the Roberts, Sobel and Prewitt operators. However for the propose of this work, we have select two detectors for comparison. These are the well-known Canny[12] and Marr Hildreth[13] edge detectors, and an application of Hough Transform. [14-16]

For evaluation of algorithms adaptations, we develop a method for automatic cell segmentation and measurement. Our goal is to find a set of parameters defining the location of the objects to compare the original and processed images.

\section{Methodology}

A general problem for edge detection is sensitivity to noise, because in the border detection it is possible to localize changes of intensity where edge doesn't exist. Therefore the degree of success of an edge detector depends on its ability to precision 
locate the true edges. Another problem comes from the sharp intensity transitions, that it indicates that the edge are shape owed of their high frequency components. As consequence, any smoothing or linear filtering are necessary since the edge detection depends on the differentiation the image function.

Although this problem is addressed in the Canny and Marr operators by convoluting the image with smoothing operator (Gaussian) before calculating the derivative it was necessary we use a medium filter to brighten up the noises.

The effect of Gaussian smoothing is to blur an image, the degree of smoothing is determined by the standard deviation, where the larger standard deviation Gaussians require larger convolution kernels in order to be accurately represented.

One of the main justifications for using the Gaussian as a smoothing filter is due to its frequency response. Most convolution -base smoothing filter act as lowpass frequency filters. This means that their effect is to remove low spatial frequency components from an image.

\section{Canny edge detector}

The Canny edge detector arises from the earlier work of Marr and Hildreth, who were concerned with modeling the early stage of human visual perception. The algorithm performs the smoothed of image by a two dimensional Gaussian function. The width is specified by a user parameter. We approximated a two dimensional convolution by two one dimensional Gaussians, one aligned with the x-axis, the other the y-axis, because two dimensional convolution with large Gaussian takes a long time. After that it is normalized the gradient, combining the $\mathrm{x}$ and $\mathrm{y}$ directional derivatives.

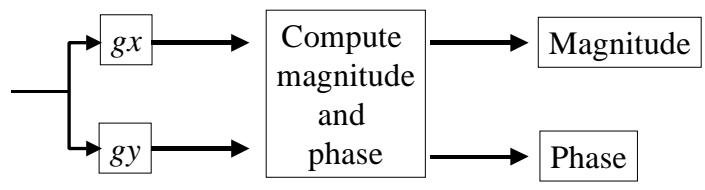

The next step is apply a tracking process where exhibits hysteresis . The hysteresis threshold serve to connect the contour points. There are two parameter, the "low threshold" and "high threshold". All points with a magnitude greater than high threshold are declared "points of contour". All points with a magnitude less than low threshold are rejected. The points that are between low threshold and high threshold are connected. The thresholds are set according to the amount of noise in the image, which is determined by a noise estimation procedure. This hysteresis helps to ensure that noisy edges are not broken up into multiples edge fragments.

Finally, apply interpolation to find the pixels where the norms of gradient are local maximal. By interpolation of the surrounding discrete grad values, the gradient magnitudes are calculated at the neighborhood boundary in both directions perpendicular to the center pixel. A local maximum occurs at a peak in the gradient function, or alternatively where the derivative of the gradient function is set to zero.

The thinning of the edge is an important step, all points that are not local maximal along a line pointing in the direction of the gradient are removed.

The problem found in this algorithm is that marks a point as an edge if its amplitude is large than that of its neighbors without checking that the differences between this point and its neighbors are higher than what is expected for random noise. That does with the algorithm is more sensitive to weak edge, and this turns it more susceptible to the false and unstable boundaries whenever is an insignificant change of the intensity

Summarizing, in Canny three optimality criteria are as follows:

\section{Good detection}

In the case of write Gaussian noise, the probability of false detection decreases with increasing signal-to-noise ratio (SNR). The SNR at an edge point is:

$$
S N R=\frac{\left|\int_{-\infty}^{\infty} G(-x) f(x) d x\right|}{\sqrt[\sigma]{\int_{-\infty}^{\infty} f^{2}(x) d x}}
$$


where $f(x)$ denotes the optimal operator, $G(x)$ denotes the edge and $n_{0}$ is the root-mean-squared value of the noise. It is assumed that the optimal filter has a finite pulse response bounded by $[-\infty, \infty]$.

\section{Good localization}

This criterion is expressed as the inverse of position variance of maximum of the filter response about the correct location. A Taylor series expansion of the filter output yields the mean-squared value of the deviation $E\left[x_{0}^{2}\right]$ :

$$
E\left[x_{0}^{2}\right]=\frac{\sigma^{2} \int_{-\infty}^{\infty} f^{\prime 2}(x) d x}{\left[\int_{-\infty}^{\infty} G^{\prime}(-x) f^{\prime}(x) d x\right]^{2}}
$$

Localization is defined as the reciprocal de $E\left[x_{0}^{2}\right]$ :

$$
\text { Localization }=\frac{\left|\int_{-\infty}^{\infty} G^{\prime}(-x) f^{\prime}(x) d x\right|}{\sqrt[\sigma]{\int_{-\infty}^{\infty} f^{\prime 2}(x) d x}}
$$

where $f^{\prime}(x)$ us the derivative of $f(x)$.

Low Multiple Response

Designed to minimize the number of spurious maximal within the operator's spatial spread. The expression for the distance between adjacent maximal of the filter output in response to noise is

$$
x_{\max }=2 \pi\left(\frac{\int_{-\infty}^{\infty} f^{\prime 2}(x) d x}{\int_{-\infty}^{\infty} f^{\prime \prime 2} d x}\right)^{\frac{1}{2}}=k w
$$

fixing the constant $k$ to be as large as possible results in the distance between spurious maximal being as great as possible. [12,17-29]

\section{Marr Hildreth edge detector}

In the seventies, the theory of Marr concluded from experiments neurophysiological that the border of the objects is the most important data to unite the image of intensities with its interpretation. The edge detectors that existed in that time were based on the convolution with very small masks and they only worked on certain images. Its main disadvantage was its dependence of the sizes of the objects and its sensibility to noises.

After this time Marr and Hildreth proposed the spatial coincidence assumption which led to the idea of multi-scale edge analysis. They observed significant intensity changes occur at different scales with an image. The conclusion was that optimal detection would requires the use of a filter that operates ate several different scale. They had concluded then that the edge maps of the different scales each contained important information about physically significant phenomena. That is to say the change of intensity of an image due to an only physically phenomena will happen when the border this present in several different scales. Even, to produce the intensity change in a same region, the border has to be present an only scale, then considered two independent physically phenomena. It is important to stand out that this supposition is based on the intuition. 
The advantaged of the Marr Hildreth detector is that it always produces closed contours. However, this method doesn't localize edges as well as the second directional derivative in regions where the edge is highly curved.

Using derivatives on a real sampled image is always sensitive due to the noise that is always present, the Marr Hildreth algorithm becomes even more sensitive because of it's use the second derivative.

The crucial point is as calculating second derivative in a robust way. A possibility is to smooth the image first (to reduce the noise) and later to calculate the second derivative. However to reduce the effects of noise the derivative is usually combined with a Gaussian filter.

Summarizing, the Marr Hildreth algorithm can be implemented in the following way:

(1) Convoluting a image with a two-dimensional Gaussian to reduce the effects of image noise. The Gaussian operator is given by

$$
g(x, y)=\frac{1}{2 \pi \sigma^{2}} \exp \left[-\left(\frac{x^{2}+y^{2}}{2 \sigma^{2}}\right)\right]
$$

(2) Compute the second derivative of the image intensities using second derivative differential operator such as the Laplacian.

(3) Identify the edges as the zero crossing in the second derivative.

The Laplacian of a Gaussian function as a filter:

$$
\begin{array}{r}
\nabla^{2} g(x, y)=\frac{d^{2}}{d x^{2}} g(x, y)+\frac{d^{2}}{d y^{2}} g(x, y) \\
\quad=\frac{x^{2}+y^{2}-2 \sigma^{2}}{2 \pi \sigma^{6}} \exp \left[-\left(\frac{x^{2}+y^{2}}{2 \sigma^{2}}\right)\right]
\end{array}
$$

This is an orientation independent operator and its scale is given by $\sigma$.

\section{Modified Marr Hildreth}

In this implementation we used ourselves a way that allows to use the information of the first derivative under a selective criterion to reject undesirable contours caused by noise.

This criterion allows that stays the contours where the gradient is " wide enough " allowing to remove the undesirable contours, while it maintains the closed contours of the original algorithm. We observed that after the experiments, we obtain better results to maintain the shut contours when the value of the magnitude gradient is larger than the threshold. We defined that the very small contours would be despised.

Therefore it can define the edges as being the zero crossing of the second derivative. The value of the magnitude gradient of a border is defined as maximum value of the magnitude gradient of two neighboring pixels and the contour is a group of all connected edges. The medium value of the magnitude of a contour defined as the average gradient magnitude on the contours. The length of a contour equals the number of edges.

It is difficult to find the number of interesting and uninteresting contours in an image because the data varies greatly with the type of image and the amount of noise.

Then it is necessary to limit the following properties:

- The maximum magnitude value of the gradient

- The length of a contour (Mostly to be combined with some other criteria)

- The minimum magnitude value of a contour

- The mean magnitude value of a contour.

The standard Marr Hildreth algorithm finds a number of good edge but even more not so good ones.

This implementation works as following:

First: Calculate the gradient magnitude value and a second derivative in every pixel. 
Second: Find the contours and their corresponding gradient magnitude values.

Third: Select which counter to keep and which to discard. The gradient magnitude and the second derivative is correct up to a constant. Then the image is filtered with a Gaussian filter. Instead of 2-D convolution, we applied equivalently two onedimensional filters along rows and column. For efficiency we calculate the Gaussian and first derivative simultaneously. [23-31,13]

\section{Hough Transform}

The Hough transform is a technique which can be used to isolate features of a particular shape within an image. Because it requires that the desired features be specified in some parametric form, the classical Hough transform is most commonly used for the detection of regular curves such as lines, circles, ellipses, etc. A generalized Hough transform can be employed in applications where a simple analytic description of a feature(s) is not possible. Due to the computational complexity of the generalized Hough algorithm, we restrict the main focus of this discussion to the classical Hough transform. Despite its domain restrictions, the classical Hough transform (hereafter referred to without the classical prefix) retains many applications, as most manufactured parts (and many anatomical parts investigated in medical imagery) contain feature boundaries which can be described by regular curves. The main advantage of the Hough transform technique is that it is tolerant of gaps in feature boundary descriptions and is relatively unaffected by image noise.

The idea of the Hough transform is to look in a parameter space what set of parameters would best fit the searched pattern in an image. We will here consider 2 simple cases, line and circle detection. A line is defined by 2 parameters, $r$ and th for example if the line equation is $\mathrm{x} \cdot \cos (\mathrm{th})+\mathrm{y} \cdot \sin (\mathrm{th})=\mathrm{r}$. th is then the angle between the line and the $\mathrm{x}$ axis while $\mathrm{r}$ is the distance from the line to the origin. The parameter space in this case is $(\mathrm{r}, \mathrm{th})$ while the 'real' (image) space is (x,y). So what we want it as function from $(\mathrm{x}, \mathrm{y})->(\mathrm{r}, \mathrm{th})$ that will give us the set $(\mathrm{r}, \mathrm{th})$ that best fit the equation of the line in the image. One way to do this would be to look for all pair of points in the image what are the value of $(r$, th) defining the line going from one point to another, add the votes for each $(\mathrm{r}, \mathrm{th})$ pair, and take the highest vote after all the pair of points have been considered. This method would require a huge computational power. The approach used in the Hough transform is to consider each 'interesting' point and look at the set of curves that could go through that point. Interesting means that a point has a much higher value than the background. So for each interesting point $(\mathrm{x}, \mathrm{y})$ one has the set of lines possibly going through this point defined as $\mathrm{r}=\mathrm{x} \cdot \cos (\mathrm{th})+\mathrm{y} \cdot \sin (\mathrm{th})$ (remember, we are now in $(\mathrm{r}, \mathrm{th})$ space while $\mathrm{x}$ and $\mathrm{y}$ are given, so we want to draw $r$ as a function of th). We sum all the votes (curves) $r(t h)$ for each pair of interesting (x,y), and at the end of the algorithm, the point in parameter space which has most votes is the set of parameters that best defines the curve.

The same is true for circles. This model has three parameters: two parameters for the centre of the circle and one parameter for the radius of the circle. If the gradient angle for the edges is available, then this provides a constraint that reduces the number of degrees of freedom and hence the required size of the parameter space. The direction of the vector from the centre of the circle to each edge point is determined by the gradient angle, leaving the value of the radius as the only unknown parameter.

Then the Hough Transform algorithm for circle fitting can be described as follows.

\section{Circle Fitting Algorithm}

1. Quantise the parameter space for the parameters $a$ and $b$.

2. Zero the accumulator array $\mathrm{M}(\mathrm{a}, \mathrm{b})$.

3. Compute the gradient magnitude $\mathrm{G}(\mathrm{x}, \mathrm{y})$ and angle $\bullet(\mathrm{x}, \mathrm{y})$.

4. For each edge point in $\mathrm{G}(\mathrm{x}, \mathrm{y})$, increment all points in the accumulator array $\mathrm{M}(\mathrm{a}, \mathrm{b})$ along the line

$\mathrm{b}=\mathrm{a} \tan \bullet-\mathrm{x} \tan \bullet+\mathrm{y}$

5. Local maximal in the accumulator array correspond to centers of circles in the image. [14-16]

\section{Measurement}

The parameters of measurement apply in this work can be described as follows[31]:

- Filled area, Taut string area, Perimeter, Ferets, Diameter and Fiber length

\section{Conclusions}

The modification proposed to the Marr Hildreth algorithm is able to remove most of the weak and uninteresting edge while keeping the closed character of the contours. 
This implementations has also inherited some of the weaknesses from original algorithm, there are:

It is necessary to apply the Gaussian filter because the use of a second derivative filter is sensitive a noise

- Some contours contain both good strong edges corresponding to interesting structure in the image and weaker parts that are caused by noise or other uninteresting weak structures. The contour is "leaking'.

\section{Advantage}

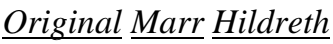

- Very fast

- Closed contours

Canny's edge detector

- Good edge detection - there should be low probabilities of failing to mark real edge points an marking false edge points.

- Good edge localization - the position of edge points marked by the edge points.

- Clear response - only response per edge.

\section{$\underline{\text { Modified }} \underline{\text { Marr }} \underline{\text { Hildreth }}$}

- Less sensitive to noise that original Marr Hildreht

- Closed edges

- Compared to Canny's edge detector these weaknes becomes obvious. By not explicitly calculating a second derivative it doesn't need the same smoothing. Canny's algorithm doesn't enforce closed contours, so it doesn't run into the problem with leaking contour either. So if closed edge are not desired Canny's edge detector is superior.

\section{Disadvantage}

\section{Original Marr $\underline{\text { Hildreth }}$}

- Lost of edges that are caused by noise and doesn't correspond to interesting features in the original image

- Sensitive to noise due to use of second derivatives

\section{$\underline{\text { Modified }} \underline{\text { Marr }} \underline{\text { Hildreth }}$}

- Sensitive to noise due to use of second derivative

- Leaking contours.

\section{Hough Transforms}

- If an image consists of objects with known shape and size, segmentation can be viewed as a problem of finding this object within an image.

- One possible way : to move a mask with an appropriate shape and size along the image and look for correlation between the image and the mask.

Unfortunately, the specified mask often differs too much from the object's representation in the processed data, because of shape distortions, rotation and zoom, among other.

\section{BIBLIOGRAPHY}

[1] D. DORI and R.M. HARALICK, "A pattern recognition approach to the detection of complex edges, Pattern recognition Letters, vol. 16, n.7, 1995, 947 - 964 
[2] D. ZIOU and S. TABBLNE, “A multi-scale edge detector”, Pattern Recognition, vol 26, no.9, 1993, 1305-1314

[3] VINCENT TORRE, TOMASO A. POGGIO, On Edge Detection, Vol. PAMI-8, n.2, 1986.

[4] BICHER. P., "Edge deteciton and geometric methods in computer vision, Ph.D dissertation, Dep. Math, Univ. California, Berkel, 1984 Image Processsing, vol.13, 1980

[5] SIUZDAK, JERZY, “A Single Filter for Edge Detection”, Pattern Recognition”, vol. 31, n 11, 1998

[6 GARRIDO, A., PÉREZ de la BLANCA, "Applying deformable templates for cell image segmentation" Pattern Recognition 33(2000) 821-832

[7] PEIXOTO, N.L.V , "Matrizes Neuro-Eletrônicas”, FAPESP, No 98/02911-9

[8] P. H. GREGSON, "Angular dispersion of edgel orientation: The basis for profile insensitive edge detection", SPIE 1607 (1991), 217-224.

[9] H . H. BAKER AND T. O. BINFORD, Depth from edge and intensity based stereo, Proceedings of the 7th International Joint Conference on Artificial Intelligence (Vancouver, Canada), August 1981, pp. 631-636.

[10] Y. OHTA AND T. KANADE, Stereo by intra and inter scanline search, Transactions on Pattern Analysis and Machine Intelligence, vol. PAMI-7, March 1985, pp. 139-154.

[11] R. M. HARALICK, Digital step edges from zero crossing of second directional derivatives, IEEE Transactions on Pattern Analysis and Machine Intelligence, vol. PAMI-6, January 1984, pp. 58-68

[12] J. F. CANNY. "A computational approach to edge detection" IEEE Trans. Pattern Analysis and Machine Intelligence, pages 679-698, 1986.

[13] MARR, D AND HILDRETH E., “Theory of Edge detection”, Proc. R.Soc. Lond. B 207, 1980

[14] CLARK F. OLSON Improving the Generalized Hough Transform Through Imperfect Grouping Image and Vision Computing, 16(9-10): 627-634, July 1998

[15] ROBERT A. MCLAUGHLIN AND MICHAEL D. ALDER The Hough Transform Versus the UpWrite, IEEE Transactions on Pattern Analysis and Machine Intelligence, Vol. 20, n. 4, April 1998 [16] A.S. AGUADO, M.E. MONTIEL AND M.S. NIXON Arbitrary shape Hough transform by invariant geometric Features In Proc. International Conference on Systems, Man and Cybernetics, IEEE SMC'97, Vol. 3/5, pp. 2661--2665 October 12-15, Florida, USA 1997 IEEE.

[17] J. F. CANNY. Finding Edges and Lines in Images. Technical Report 720, MIT Artificial Intelligence Laboratory, Dept. of Electrical Engineering and Computer Science, M.I.T., Cambridge Mass., 1983. [18] I.T. LAANCASTER and D.G. ELLIMAN, "A Comparison of two algorithms for threshold using edge detection techniques", Pattern Recognition Letter, vol.11, n. 3, 1990, 175-180 [19] DEMIGNY, DIDIER; KAMLE, TAWFIK "Discrete expression of Canny's criteria for step edge detector performances evaluation" IEEE Transactions on Pattern Analysis and Machine Intelligence

Volume 19, Issue 11 November 1997 Pages 1199-1211

[20] CENTENO, JORGE A. SILVA AND HAERTEL, VICTOR, "An Adaptive Image Enhancement Algorithm”, Pattern Recognition, vol. 30, n 7, 1997

[21] PAKER, J.R,.. "Gray Level Thresholding in Badly Illuminated Images", IEEE Transactions on Pattern Analysis and Machine Intelligence, vol 13, 1990

[22] GUNN, STEVE R., "On the discrete representation of the Laplacian of Gaussian" ., Pattern Recognition 32(1999) 1463-1472

[23] LESA M. KENNEDY and MITRA BASU, "Image enhancement using a human visual system model" , Pattern Recognition, vol. 30, n 12, pp. 2001-2014, 1997

[24] SUDEEP SARKAR and KIM L. BOYER, “On Optimal Infinite Impulse Response Edge Detection Filters" , IEEE Transactions on Pattern Analysis and Machine Intelligence, vol. 13. n 11, November 1991

[25] JACH KOPLOWITS and VITO GRECO, "On the Edge location Error for Local Maximun and Zero-Crossing Edge Detectors" , IEEE Transactions on Pattern Analysis and Machine Intelligence, vol.16 n0. 12, December 1994

[26] HEMAND D. TAGARE and RUI J.P. FIGUEIREDO, "On the Localization Performance Measure and Optimal Edge Detection”, IEEE Transactions on Pattern Analysis and Machine Intelligence, vol. 12, n 12, December 1990

[27] KIM L. BOYER and SUDEEP SAKAR , "On the Localization Performance Measure and Optimal Edge Detection", IEEE Transactions on Pattern Analysis and Machine Intelligence, vol 16, n. 1, January, 1994

[28] AMLAN KUNDU, “Robust Edge Detection”, Pattern Recognition, vol. 23, n 5, pp. 423-440, 1990

[29] STEVE R. GUNN, "On the discrete representation of the Laplacian of Gaussian", Pattern Recognition 32 (1999) 1463-1472

[30] GONZALEZ, RAFAEL C. AND WOODS, RICHARD E., "Digital Image Processing”, Ed. Addison , Wesley Publishing Company, 1992

[31] JOHN C. RUSS, The Image Processing Handbook, Boca Raton, Fla. : CRC Press, c1992 